\title{
Evaluación de la deformación miocárdica de ventrículo derecho en tetralogía de Fallot corregida quirúrgicamente
}

Julio Cesar Biancolini'

Fernanda M. Biancolini

Ana M. S. de Dios ${ }^{2}$
Correspondencia

Julio César Biancolini

biancolini@gmail.com

'Médico de planta, unidad de Cardiología Infantil, Hospital General de Niños "Dr. Pedro de Elizalde", Buenos Aires, Argentina.

`Jefa de Unidad de Cardiología Infantil, Sanatorio Trinidad Mitre, Buenos Aires, Argentina.

Recibido: 16/10/2021

Aceptado: 17/10/2021

En línea: 15/12/2021

Citar como: Biancolini J, Biancolini F, De Dios A.: Evaluación de la deformación miocárdica de ventrículo derecho en tetralogía de Fallot corregida quirúrgicamente. RETIC. 2021 (Diciembre); 4 (3): 56-58. doi: 10.37615/retic.v4n3a16

Cite this as: Biancolini J, Biancolini F, De Dios A.: Evaluation of right ventricle strain in Fallot's Tetralogy surgically corrected. RETIC. 2021(Diciembre); 4 (3): 56-58. doi: $10.37615 /$ retic.v4n3a16

\section{Palabras clave}

$\triangleright$ Tetralogía de Fallot

$\triangleright$ Deformación miocárdica.

$\triangleright$ Cardiopatía congénita.

\section{Keywords}

$\triangleright$ Tetralogy of Fallot.

$\triangleright$ Myocardial deformation.

$\triangleright$ Congenital heart disease.

\section{RESUMEN}

En pacientes con tetralogía de Fallot operada la afectación de la función sisto-diastólica del ventrículo derecho (VD) continúa siendo una complicación relevante. Atribuido a la dilatación del VD, secundaria a insuficiencia pulmonar y/o estenosis crónica, disincronía electromecánica y fibrosis miocárdica. Una adecuada valoración con técnicas de deformación miocárdica podría contribuir para indicación oportuna de reemplazo valvular pulmonar.

\begin{abstract}
In patients with repaired tetralogy of Fallot, dysfunction of the right ventricle (RV) remains an important issue Adverse right ventricular remodeling has been related to RV dilation secondary to pulmonary regurgitation, electromechanical dyssynchrony, and myocardial fibrosis. There is a growing body of literature on the use of myocardial deformation imaging in the assessment of ventricular mechanics and its clinical and prognostic values in determining the optimal timing of pulmonary valve replacement.
\end{abstract}

\section{Introducción}

La tetralogía de Fallot (TF) es la cardiopatía congénita cianótica más frecuente. La reparación quirúrgica temprana ha cambiado su pronóstico y evolución natural. Sin embargo, las complicaciones tardías son frecuentes, principalmente, la disfunción del tracto de salida ventricular derecho (TSVD), con insuficiencia pulmonar, estenosis pulmonar o ambas, la disincronía electromecánica relacionada con el bloqueo de rama derecha y diversos grados de fibrosis miocárdica, con la consecuente disfunción del ventrículo derecho (VD)'.

\section{Deformación miocárdica del ventrículo derecho}

La evaluación de la función del VD continua siendo una de las tareas más desafiantes y técnicamente dificultosas de la ecocardiografía. En los últimos años, las imágenes de deformación miocárdica ventricular derecha, han surgido como un método superador, en la valoración de la función sistólica, a los parámetros ecocardiográficos convencionales

La deformación miocárdica puede obtenerse a través de la utilización del speckle-tracking (ST) o derivado del Doppler tisular. Las ventajas del ST se basan en la independencia de la técnica del ángulo de insonación y mejor reproducibilidad.
Para su adquisición, se recomienda la vista de 4 cámaras con foco en $\mathrm{VD}^{2}$ (Tabla 1, Videos 1 y 2 ).

Al reportar la deformación miocárdica del VD se debe realizar la distinción del los segmentos incluidos en el análisis ya que la valoración de la pared libre (3 segmentos) tiene valores de referencia mayores que al incluir el septum interventricular (6 segmentos) (Videos 3 y 4 ).

En conjunto, a pesar de la heterogeneidad de los estudios con respecto a la modalidad de imagen utilizada y la evaluación regional versus global de la deformación del VD, la evidencia hasta la fecha ha demostrado en general un deterioro de la deformación miocárdica sistólica y diastólica del VD en niños y adultos después de la reparación de TF (Video 5).

El empleo del valor de corte de -17\% para la deformación longitudinal global de la pared libre del VD derivado de ST y de -24\% para la deformación global del VD derivada de ST tridimensional, permite la predicción de la disminución de la fracción de eyección del VD?3.

\section{Reemplazo valvular pulmonar}

Es de vital importancia realizar el reemplazo valvular pulmonar (RVP) o el alivio de la estenosis pulmonar antes de que la disfunción del VD sea irreversible, 


\section{- Trukipedia Truco 03}

sin embargo, determinar el momento óptimo para el RVP continúa siendo un desafío.

Las guías vigentes recomiendan el RVP para los pacientes sintomáticos con disfunción del TSVD tras su reparación, en presencia de insuficiencia pulmonar moderada a severa o estenosis pulmonar grave ${ }^{4-5}$

Las indicaciones para la restauración de la funcionalidad del TSVD post reparación quirúrgica alejada de la TF, en pacientes asintomáticos, son controvertidas. Algunos autores han demostrado que la deformación de la pared libre del VD es un predictor independiente de baja capacidad funcional en la TF reparada con insuficiencia pulmonar de moderada a grave. Un valor mayor a -17\% podría ser de utilidad para decidir cuándo realizar el reemplazo de la válvula pulmonar, cuando la capacidad funcional no pueda medirse objetivamente ${ }^{1-3}$. No obstante, se requieren estudios adicionales para evaluar la utilidad de las imágenes de deformación del VD para determinar el momento optimo para el RVP.

\begin{tabular}{|l|}
\hline $\begin{array}{l}\text { Se obtiene desde una posición más lateral del transductor que la requerido } \\
\text { para una vista estándar apical de cuatro cámaras. }\end{array}$ \\
\hline $\begin{array}{l}\text { Colocar el ápex en el centro de la imagen, mostrando el VD en su mayor } \\
\text { dimensión (longitudinal y transversal). }\end{array}$ \\
\hline Debe observarse el ápex y la pared libre del VD en toda su extensión. \\
\hline $\begin{array}{l}\text { No debe observarse la válvula aórtica (corte demasiado anterior), ni el seno } \\
\text { coronario (corte demasiado posterior). }\end{array}$ \\
\hline $\begin{array}{l}\text { La región de interés debe incluir tanto la pared libre del VD como el septum, } \\
\text { y ajustarse al ancho de la pared libre. }\end{array}$ \\
\hline $\begin{array}{l}\text { Debe evitarse colocar el área de interés sobre el pericardio (tiende a } \\
\text { subestimar). }\end{array}$ \\
\hline Colocar la muestra por debajo del anillo tricuspídeo. \\
\hline Algunos programas, solo le pedirán delinear el endocardio ventricular. \\
\hline
\end{tabular}

Tabla 1. Consejos para la adquisición adecuada y procesado de

deformación miocárdica de VD.

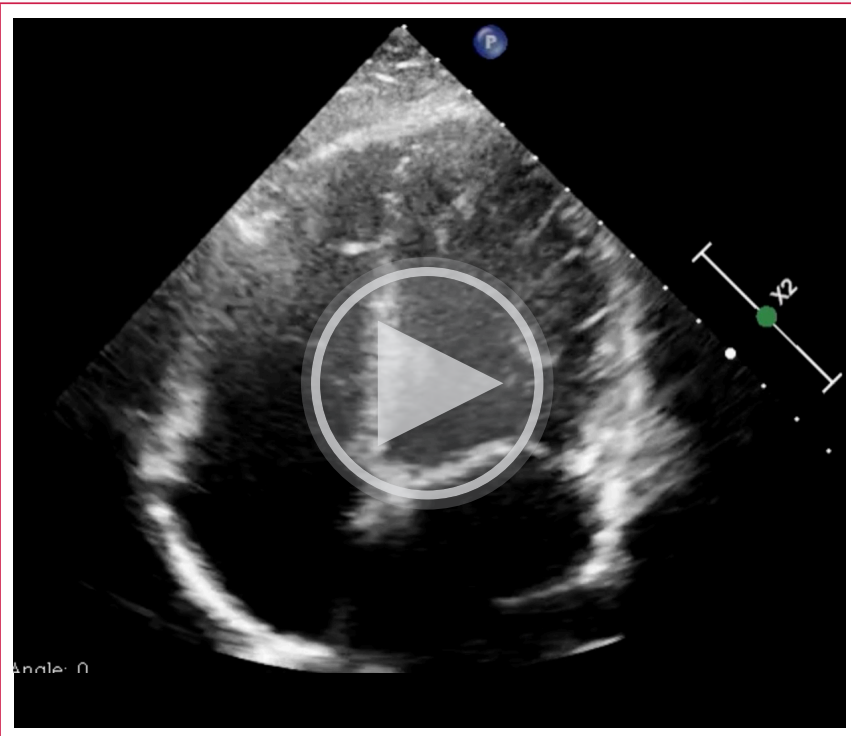

Vídeo 1. Ecocardiograma transtorácico, vista de 4 cámaras con foco en VD.

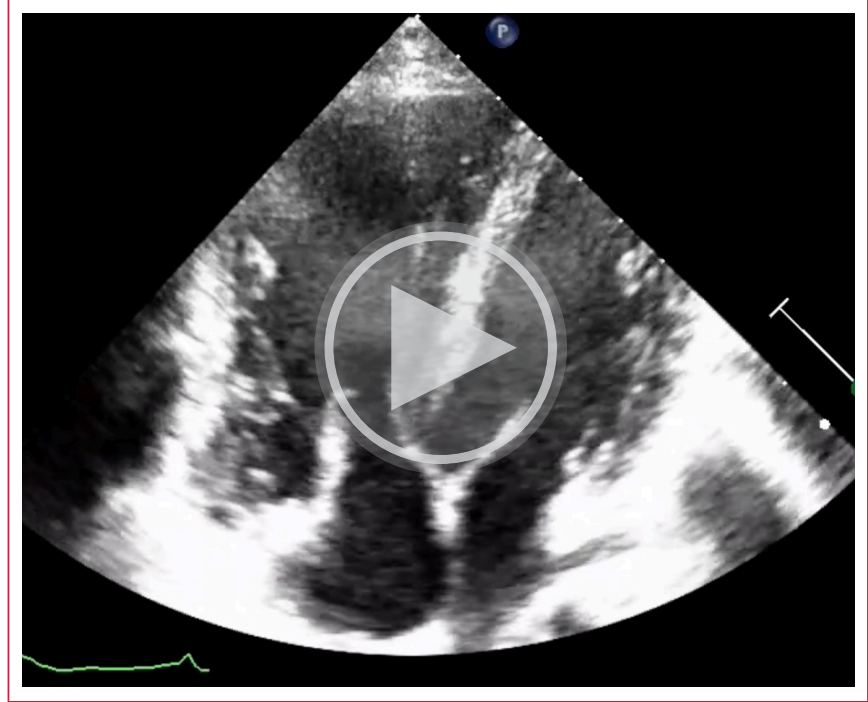

Vídeo 2. Ecocardiograma transtorácico, vista de 4 cámaras con foco en VD en TF corregida.

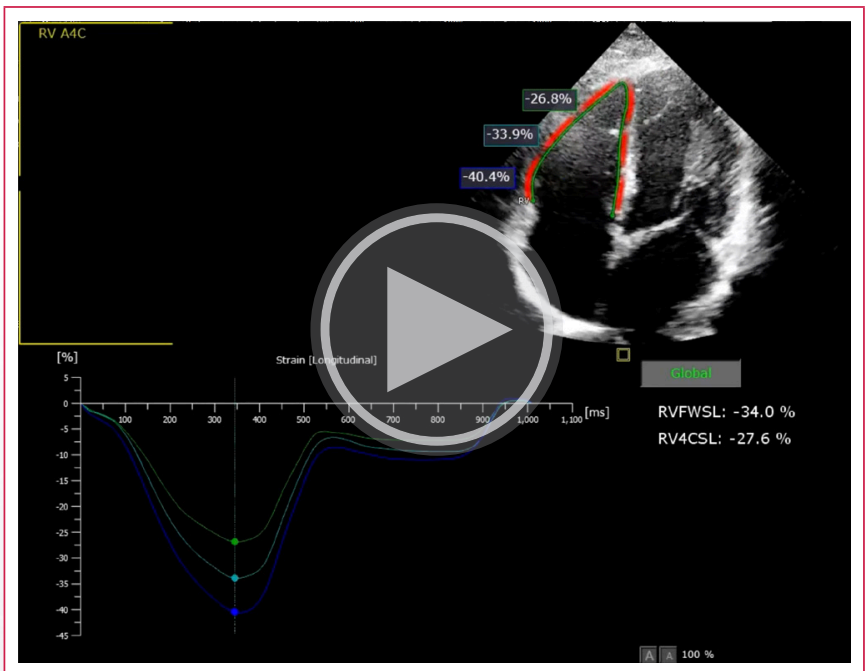

Vídeo 3. Strain de ventrículo derecho en un paciente normal: strain global $-28 \%$, strain de pared libre $-34 \%$

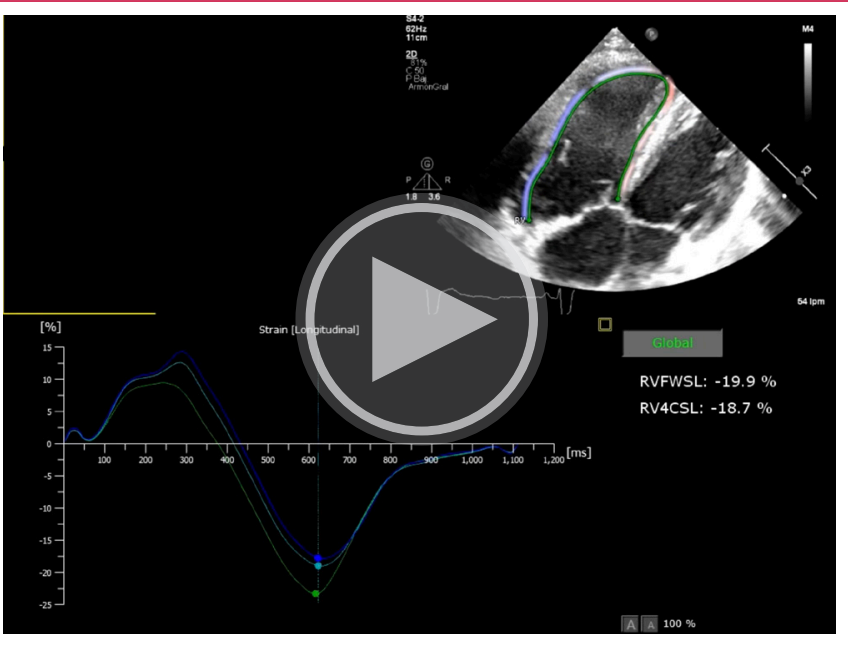

Vídeo 4. Strain de ventrículo drecho en un paciente operado de tetralogía de Fallot, strain global $-18.7 \%$, strain de pared libre $-19.9 \%$. 


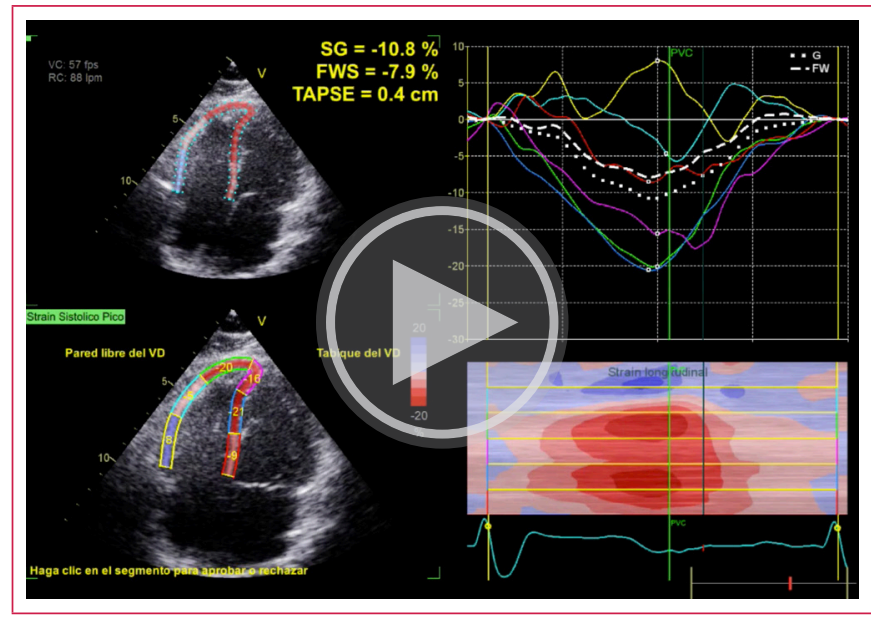

Vídeo 5 . Severa reducción de strain y disincronía en paciente operado de Tetralogía de FAllot.

\section{Ideas para recordar}

- La afectación de la deformación miocárdica del VD permitiría anticipar la disminución de la fracción de eyección del VD.

- Un valor mayor a -17\% podría ser útil para decidir cuándo realizar el RVP.

\section{Bibliografía}

1. Arroyo-Rodríguez C., Fritche-Salazar J., Posada-Martínez E., et al. Right ventricular free wall strain predicts functional capacity in patients with repaired Tetralogy of Fallot. The International Journal of Cardiovascular Imaging, 2020, 36(4):595-604.

2. Badano L., Muraru D., Parati G., et al. How to do right ventricular strain. European Heart Journal - Cardiovascular Imaging, 2020, 21, 825-827.

3. Wing-yi Li V., Kwong-man Yu C., Kam-fung So E., et al. Ventricular Myocardial Deformation Imaging of Patients with Repaired Tetralogy of Fallot. Journal of the American Society of Echocardiography, 2020; 33:788-801.

4. Torres-Alba F., Kaleschke G., Baumgartner H. Impacto del implante percutáneo de válvula pulmonar en cuanto al momento de reintervenir por disfunción del tracto de salida del ventrículo derecho. Revista Española de Cardiología, 2018;71(10):838-846.

5. Baumgartner H., De Backer J., Babu-Narayan S. 2020 ESC Guidelines for the management of adult congenital heart disease. European Heart Journal, $2020,00,1-83$ 